\title{
The Effect of Camera Height, Actor Behavior, and Viewer Position on the User Experience of $360^{\circ}$ Videos
}

\author{
Tuuli Keskinen', Ville Mäkelä2,1, Pekka Kallioniemi' ${ }^{1}$, Jaakko Hakulinen'1, \\ Jussi Karhu1', Kimmo Ronkainen', John Mäkelä', Markku Turunen ${ }^{1}$ \\ Tampere University, Finland ${ }^{1}$ \\ LMU Munich, Germany²
}
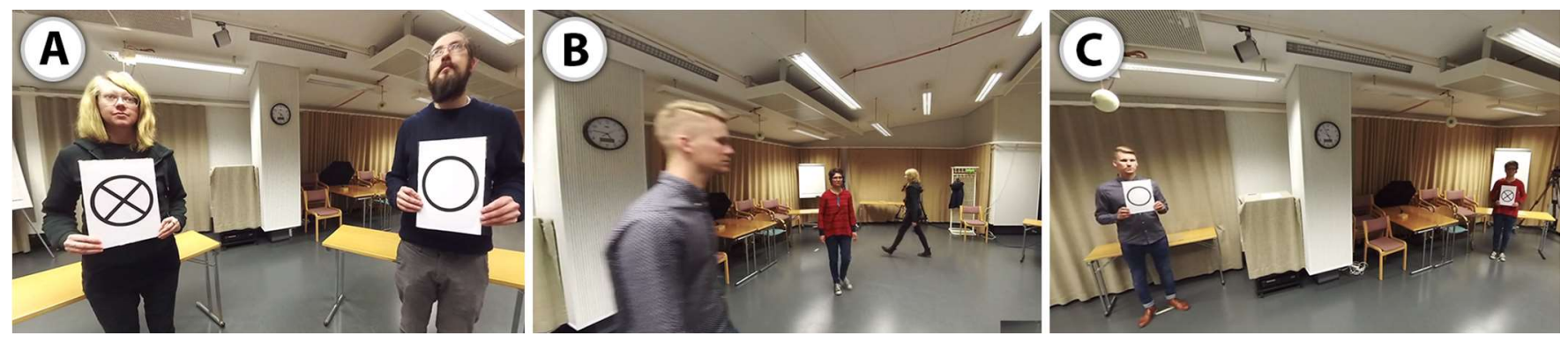

Figure 1: Examples of the evaluated $360^{\circ}$ scenes with varying camera heights and actor behavior. A: Actors standing near the camera, camera height $132 \mathrm{~cm}$. B: Actors moving around, camera height $159 \mathrm{~cm}$. C: Actors standing far from the camera, camera height $185 \mathrm{~cm}$.

\begin{abstract}
$360^{\circ}$ videos can be viewed in an immersive manner with a headmounted display (HMD). However, it is unclear how the viewing experience is affected by basic properties of $360^{\circ}$ videos, such as how high they are recorded from, and whether there are people close to the camera. We conducted a 24-participant user study where we explored whether the viewing experience is affected by A) camera height, B) the proximity and actions of people appearing in the videos, and C) viewer position (standing/sitting). The results, surprisingly, suggest that the viewer's own height has little to no effect on the preferred camera height and the experience. The most optimal camera height situates at around 150 centimeters, which hits the comfortable height range for both sitting and standing viewers. Moreover, in some cases, people being close to the camera, or the camera being very low, has a negative effect on the experience. Our work contributes to understanding and designing immersive $360^{\circ}$ experiences.
\end{abstract}

Keywords: $360^{\circ}$ videos, camera height, omnidirectional videos, head-mounted displays, virtual environments, virtual reality, user experience, viewer height, viewer position.

Index Terms: Human-centered computing_Virtual reality

\section{INTRODUCTION}

$360^{\circ}$ videos - or omnidirectional videos (ODVs) - are a new type of medium that capture an environment in its entirety. Such videos

\footnotetext{
1 tuuli.keskinen, ville.makela, pekka.kallioniemi, jaakko.hakulinen, jussi.karhu, kimmo.ronkainen, john.makela, markku.turunen\}@tuni.fi

2 ville.maekelae@ifi.lmu.de (work reported in this paper was done while with Tampere University)
}

are optimally viewed by using a head-mounted display (HMD), wherein the video is set in a spherical view around the user, and by utilizing the head-tracking features of HMDs, the viewer can observe the video content naturally by turning their head.

Using an HMD is an immersive and enjoyable way of viewing $360^{\circ}$ videos [10][14]. Such setups have already been used in countless contexts, such as collaborative wayfinding activities [9], industrial maintenance tasks [20], cinematic virtual reality [4][18][19], and recreational simulator experiences [6][20].

However, despite the recent interest towards $360^{\circ}$ videos and HMDs, many basic matters regarding the viewing experience and user preferences in $360^{\circ}$ videos are unexplored. In this paper, we especially focus on the theme of viewer and camera height, and actor behavior, that is, the height of the viewer, the virtual height of the camera, and the proximity and actions of the people appearing in the videos (Figure 1). For instance, it is unclear whether the viewing experience is affected by differences between the viewer's actual height and the height from which the video is recorded. Similarly, it is unclear whether the actions and proximity of the people in the videos affect the viewer's experience. These characteristics are fundamental in many types of $360^{\circ}$ content, and therefore present an important research topic.

With these research gaps in mind, we investigate the following research questions:

- How does camera height in $360^{\circ}$ videos affect the viewing experience and what is a comfortable camera height range?

- How do the proximity and actions of people in $360^{\circ}$ videos affect the viewing experience?

- How do different viewing positions, sitting and standing, affect the viewer experience in relation to camera height and actor proximity?

To explore these questions, we conducted a 24-participant user study that consisted of two parts. In the first part, the participants performed simple search tasks while watching $360^{\circ}$ videos, wherein we varied the camera height as well as what the people in the videos were doing. We collected subjective feedback from the conditions and also logged the participants' gaze data. In the second part, the participants viewed, and were able to browse 
between, $360^{\circ}$ videos recorded from 16 different camera heights, and reported their most comfortable, the highest still comfortable, and the lowest still comfortable camera heights for both sitting and standing positions.

Our results show that the viewer's own height has no effect on what camera height(s) they prefer, and very little effect on the viewing experience with different camera heights. Sitting viewers prefer slightly lower viewpoints than standing viewers. However, by placing the camera at around $150 \mathrm{~cm}$, the comfortable height range can be hit for both types of viewers. This height also aligns with our viewing experience results, as the cameras within the $\sim 130-160 \mathrm{~cm}$ height range resulted in a better experience than cameras outside this range. Interestingly, the most liked heights in this study are lower than the eye level of most people.

Moreover, the viewing experience was evaluated slightly worse in cases where people in the $360^{\circ}$ videos were close to the camera. It is therefore advisable to leave room between the camera and the people and other objects in the videos. These results predict that the traditional rules of personal space and proxemics [7] may be applicable in the context of $360^{\circ}$ videos. The viewing experience was also evaluated to be slightly worse when the camera was very low or very high.

Our results are helpful in understanding and designing immersive $360^{\circ}$ experiences, particularly through our three main results. First, we show that viewer's height has no meaningful effect on the viewing of $360^{\circ}$ videos, and the most natural and preferred viewpoints are lower than an average viewer's realworld viewpoint. Second, while sitting viewers tend to prefer somewhat lower viewpoints, the comfortable range is large enough to accommodate for both viewing positions with one camera height. Third, very low and very high camera heights as well as people close to the camera should be avoided whenever possible, as they may harm the viewing experience.

\section{Related Work}

\section{$2.1360^{\circ}$ Videos}

$360^{\circ}$ videos have been studied extensively in the recent years, and when combined together with HMDs, they have been reported to be an immersive medium that provides the user an experience with a high sense of presence [4][6][9][10]. Many factors affect the user experience of $360^{\circ}$ videos, including gender and production values [9], and viewing platform [10][17][21]. Argyriou et al. [1] and Saarinen et al. [20] also present a number of recommendations for omnidirectional video applications, which we discuss in more detail in the following sections.

In this paper, the terms $360^{\circ}$ video and omnidirectional video (ODV) are used interchangeably. When considering the previous work on this topic, both of these terms are being used. The term ODV is used mostly in academic contexts, whereas $360^{\circ}$ video is used more in the mainstream contexts, including most streaming services that support this medium. Occasionally, the term panoramic video is also used [17]. In addition, the term iODV has been defined for applications that allow some sort of interaction with the content [9][10][20]. To be clear, though, in the experiment described in this paper, participants did not interact with the videos beyond looking around with the HMD.

\subsection{Camera Height and Position}

We are aware of only one study that investigated the effect of camera height in $360^{\circ}$ videos and HMDs: in their recent short paper, Rothe et al. [19] found that users generally preferred lower camera heights than higher camera heights, relative to their own height. However, in our paper we engage in a more comprehensive study of camera heights, and also supplement it with variations in the $360^{\circ}$ content. Saarinen et al. [20], on the other hand, suggest filming $360^{\circ}$ videos at a realistic camera height when it is important to show the environment as it would appear if the person was really there. This would be the case, for instance, when ODVs are used to familiarize the viewer with a location before actually going there. However, it is unclear if this recommendation holds from the user experience point of view.

Of relevance is also the perception of self within a $360^{\circ}$ video. Passmore et al. [16] and Philpot et al. [17] concluded that this perception is affected by the position of the camera and its relative position to other objects and/or people within the scene. While watching traditional videos from a flat screen, some participants reported that they felt taller when the camera was above the average person's head height, and shorter when the camera was at lower height than this [17]. Passmore et al. [16] further noted that when watching $360^{\circ}$ videos with an HMD, viewers can experience them through both first- and third-person perspectives, depending on the camera location within the scene. In their study, participants reported feeling like 'a fly on the wall' when the camera was located up by the wall, and feeling 'two inches tall' when the camera was located between an actor and the manipulated object within the scene.

The effects of camera position and angle have also been studied in the domain of film research in various experiment settings, often related to psychological factors, such as perceived social status, attractiveness, and trustworthiness (e.g., [3] and [15]). Camera angles have also been detected to affect dominance in video-mediated communication [8]. With still images, camera angle has been found to influence comprehension and retention of events [11]. In our study, the participants could explore the $360^{\circ}$ videos freely allowing them to choose the vertical angle to look from. Therefore, the camera height itself was adjusted.

\subsection{Actor Proximity and Behavior}

First research on personal space and interpersonal distance was introduced by Hall [7] in the end of the 1950's. He developed a proxemics model that divided the interpersonal space into four categories: intimate, personal, social, and public space. Based on Hall's study, people maintain a "buffer space" around themselves, and this can be affected by certain conditions, such as nonverbal communication. This same model has also been evaluated in virtual environments (VEs) [2]. The benefit of using VEs for these types of studies is that VEs allow the realism of a field study without losing the controlled environment of a laboratory [13]. In this study, we also inspect Hall's proxemics model in the context of $360^{\circ}$ videos.

In presenting recommendations for interactive $360^{\circ}$ video applications, Saarinen et al. [20] recommend avoiding objects very close to the camera because they may feel intrusive to the viewer. Although Saarinen et al. talk about any objects in general, they use an example of people, stating that people who linger around the camera and look straight at it may make the user feel uncomfortable.

Bailenson et al. [2] studied nonverbal exchanges between participants and embodied agents within virtual environments. They suggested that even when interacting with a 3-dimensional model agent within the VE, people act as in the real world when confronted by another person; they avoid direct contact with the agent, and respect its "personal space" like in real-world situations. The presence of this agent also affected a memory task (encoding of names and numbers) given to the participants, making it more difficult for the participants. These effects were not present when the 3-dimensional humanoid model was replaced with a cylinder. 


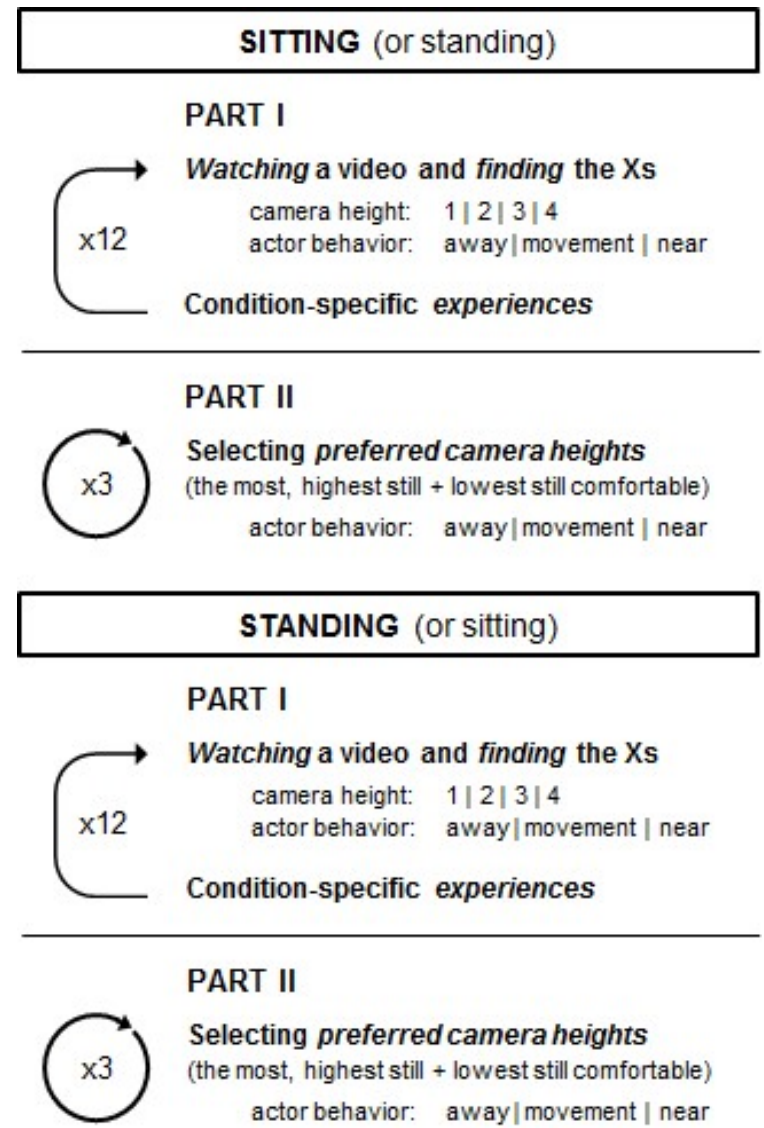

Figure 2: Experiment procedure.

\section{EXPERIMENT}

To investigate whether camera height, people's proximity and actions in the video, or the viewer's position affect the viewing experience, and to find out the optimal range for camera height, we conducted a within-subjects experiment. We recruited 24 participants to view and evaluate $360^{\circ}$ videos using an HMD.

Our experiment consisted of two main parts. In Part I, we conducted trials where two parameters were varied to investigate their effect on the viewing experience: 1) camera height (four different heights), and 2) actor proximity and behavior (three scenarios, described later in this chapter). For this, 12 different $360^{\circ}$ videos were recorded. In each video, the study participants' task was to locate a paper displaying a cross inside a circle (two times), always held by one of the actors (Figure 1A).

In Part II, we investigated the optimal camera heights in more detail. For this, we recorded a total of 48 videos with three different scenarios and 16 different camera heights. These were then used by the participants to select the camera height range they thought was comfortable.

Both parts (I \& II) were completed twice: once while sitting, and once while standing. This parameter is referred to as viewer position and was counter-balanced as were the camera heights and actor proximity and behavior. Both sitting and standing were included in the study, because they are the most common stances when using HMDs. In addition, including both leads to more comprehensive and reliable results.

The overview of this procedure is presented in Figure 2. In the following, we describe the two main parts in detail, after which we describe the full study procedure as well as the participants.

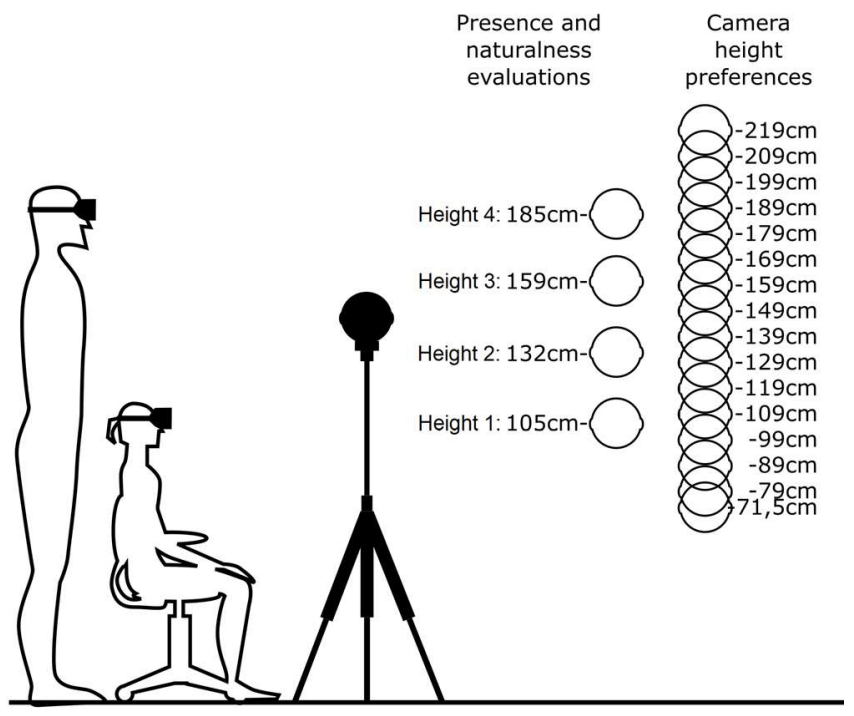

Figure 3: Camera heights used in the study. In Part I, four camera heights were used to evaluate the presence and naturalness of $360^{\circ}$ videos. In part II, participants chose their preferred height range from 16 available heights in varying scenarios.

\subsection{Part I: Viewing Experience}

In Part I, participants watched $360^{\circ}$ videos with the HTC Vive (with Tobii eye tracking) and evaluated the naturalness and the level of presence of the experience. The task in each video was to find a cross within a circle on a paper, shown by one of the actors. The participants pressed a button on the HTC Vive controller when they located the cross. This task was repeated twice in each video. For this phase, we included two independent variables:

- IV1: Camera height. Height of the camera, and therefore, the height from which the user observed the environment There were 4 levels: $105 \mathrm{~cm}, 132 \mathrm{~cm}, 159 \mathrm{~cm}$, and $185 \mathrm{~cm}$, measured from ground to the center of lenses (see Figure 3: Presence and naturalness evaluations). The lowest and highest values were based on two example persons: The shorter one, a female, sitting and the taller one, a male, standing. The two heights in between were added at equally distant points.

- IV2: Actor proximity and behavior. The distance of the actors to the camera, and their behavior. Here, we used 3 levels: away, movement, and near. The overview for the scenarios is presented in Figure 4 and the details are described below.

The two independent variables led to a total of 12 different combinations, and hence, 12 videos were recorded. In other words, all three scenarios (IV2) were recorded four times each, once for each camera height (IV1). The three scenarios (Figure 4) were as follows:

- Away condition (Figure 1C). The actors stood still, with their backs against the tables, facing the camera for about 5 seconds. Then, they took a paper from the pile on the table, showed it to the camera for about 5 seconds, and put it back. This process was repeated two times per video. The distance between the camera and the actors was 3-4 meters.

- Movement condition (Figure 1B). Here, the actors switched places. Two of the actors walked to the nearest table, while two walked to the farthest table, passing by the camera. Once arriving at the tables, the actors again took a paper from the table and showed it to the camera. This process was repeated twice. 


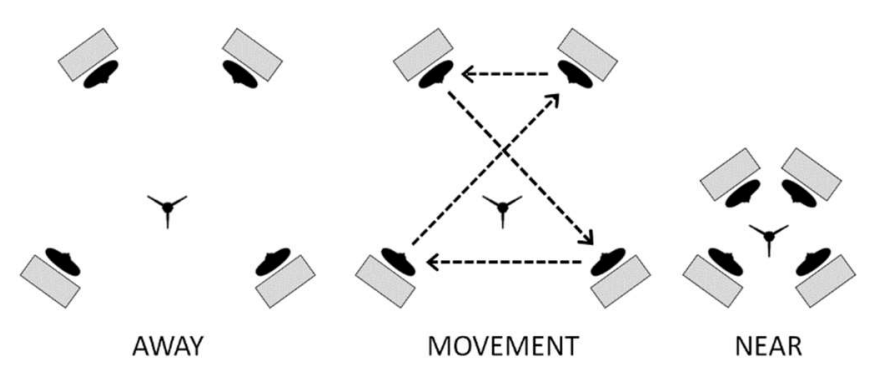

Table

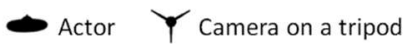

Figure 4: Actor positions and behavior: away, movement, and near.

- Near condition (Figure 1A). Here, the actors behaved exactly as in the away condition. Only the distance between the camera and the actors was smaller, about one meter.

The $360^{\circ}$ videos were recorded with an Insta360 Pro camera and an adjustable tripod. Each video displayed the tables and the four actors ( 2 females, 2 males, $163-184 \mathrm{~cm}$ tall). On the tables, there were piles of papers, from which the actors would pick up one paper at a time and show it to the camera, at which point the participant's task was to locate the paper displaying a cross inside a circle (Figure 1A). We added the search tasks for this part primarily because it gave viewers a motive to focus and look around the scenes. We also hypothesized that without a task, the scenes might feel awkward if the actors were standing and facing the camera for no apparent reason.

\subsection{Part II: Camera Height Preferences}

In Part II, the participants viewed similar scenarios from Part I (away, movement, near) and chose their preferred camera heights for each. This time, there was no search task, so no papers or crosses were included. Otherwise, the actors behaved similarly as in the first part, that is, simply stood still near or away, or walked to different positions.

To make the camera height selections comprehensive, we increased the number of camera heights to 16 (Figure 3: Camera height preferences). Thus, we recorded a total of 48 videos for this part, each scenario being recorded 16 times, once at each camera height. For the highest camera height, we selected a value clearly above the assumed maximum participant height, $219 \mathrm{~cm}$, and lowered the camera by $10 \mathrm{~cm}$ until $79 \mathrm{~cm}$, and the lowest recording height was $71.5 \mathrm{~cm}$, which was the lowest possible height for our equipment.

When viewing any of the three scenarios, the participants were able to move between the 16 camera heights as they pleased, using the HTC Vive controller. The duration and actor positions and movements within the videos were synced so that changing the video seemed like users were simply moving up and down in the same video.

For each scenario, the participants chose the height that they felt was the most comfortable. The participants also chose the lowest and the highest camera heights that they felt were still comfortable to them. Thus, the participants chose a comfortable viewpoint range, with the most comfortable height being somewhere inside the range. The participants made these selections by navigating to the desired height and announcing it to the experiment conductor, who then logged the selection. In this part, the videos were looped, in case the participants stayed in one condition long enough.

\subsection{Procedure and Data Collection}

In the beginning of the session, the participants were given a brief overview of the study. They then filled in an informed consent and an online background information questionnaire. Then, the HTC Vive and its controller were introduced to the participants. They put the Vive on and adjusted it so that it was comfortable, after which a brief eye-tracking calibration was performed in order to log the participants' gaze. Before starting the trials, the participants had a chance to try out moving their head and body, i.e., practice exploring the VR environment, with a non-interactive park scenery video.

Participants then went through Part I of the experiment as explained earlier. Half of the participants conducted this part sitting, and the other half standing. In Part I, the participants performed search tasks in a set of $360^{\circ}$ videos. When they located a paper displaying a cross in the video, they pressed the controller's button. The order of the conditions was counter-balanced.

After each video, subjective feedback was collected verbally (and marked down by the experiment conductor) so that the HMD could be kept on and thus save time. If the participants felt uncomfortable, they were allowed to remove the headset, though. Two user experience statements were read to the participants one by one, and the participants gave their answer as a number from 1 to 7 based on how much they agreed with the statement. While collecting the feedback, a 7-point scale figure was shown in the HMD view, indicating that 1 corresponds to "Totally disagree", 4 to "Neither agree nor disagree", and 7 to "Totally agree". The two statements were:

- Watching the people felt natural.

- I felt like I was actually in the space.

When the participants had completed Part I, i.e., all 12 videos, they moved to Part II of the study. In Part II, the participants selected three different camera heights: the most comfortable, the highest still comfortable, and the lowest still comfortable height. This was done for all three actor behavior conditions (away, movement, near). In each condition, the participants could freely move between the 16 heights recorded for that condition using the controller. The order of the conditions was counterbalanced.

After completing Part II, participants then went through both parts (I \& II) once more. In the second round, participants changed the viewing position: those that completed the first round sitting, now performed the second round standing, and vice versa.

When both rounds were complete, i.e., after both parts I and II were completed twice, the participants were given a certificate of their participation and they were free to leave. The experiment usually took a little less than an hour in total. All material as well as verbal discussions during the sessions were in English.

\subsection{Participants}

We recruited 24 participants (18 males, 6 females) from an introductory university course on interactive technology. Participating in a test conducted in our research center and writing a short report was mandatory for these students, and thus, they received no extra compensation for their participation. Requirements for participation were that one should be able to stand and use a controller with their hands. Additionally, adequate English skills were required as the experiment was to be conducted in English. 

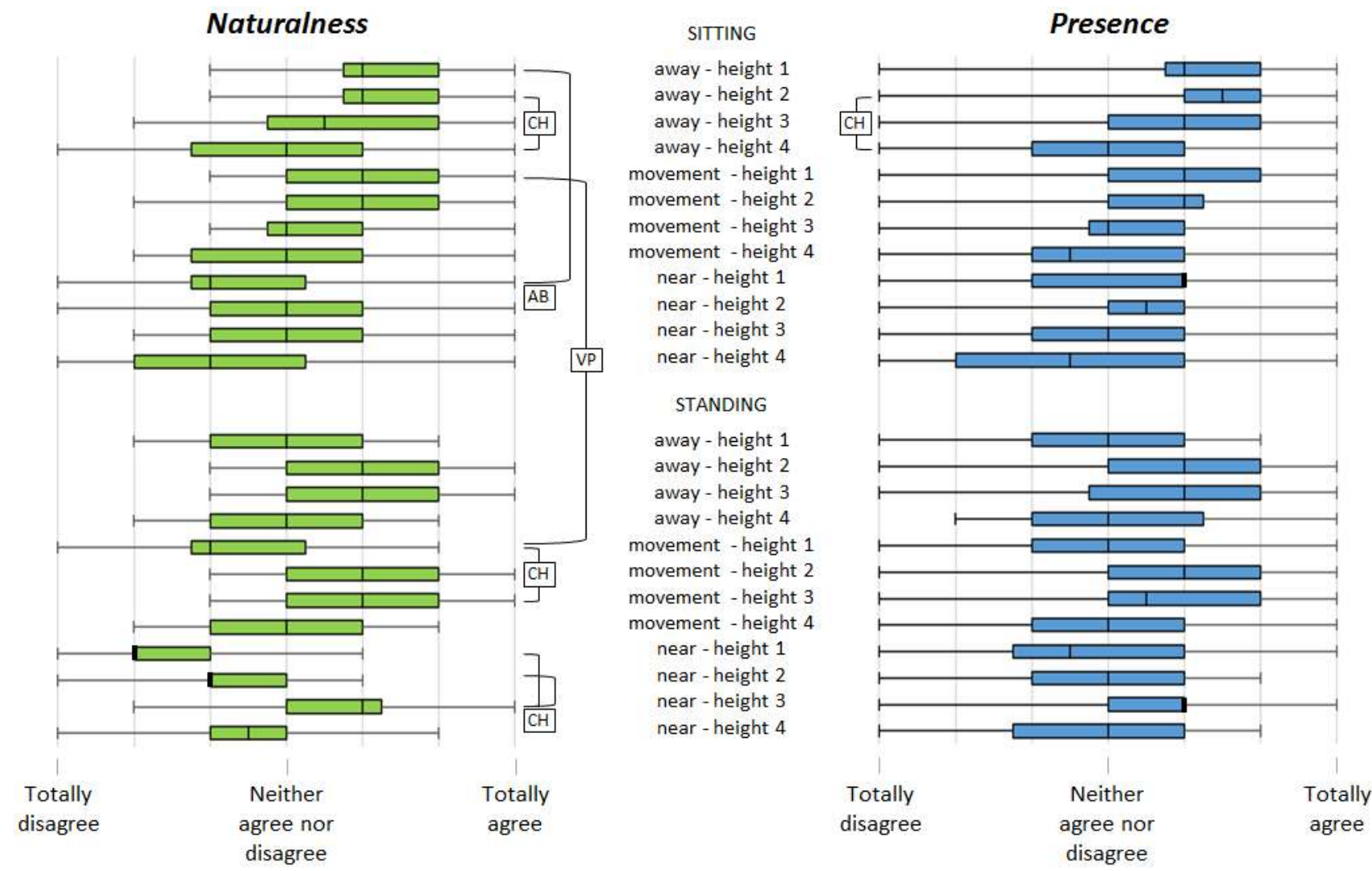

Figure 5: Boxplot presentations of the participants' assessments $(n=24)$ on naturalness of watching the people in the videos (left), and the level of presence, i.e., how highly they felt like actually being in the space (right) regarding the tested conditions. The whiskers represent the minimum and maximum ratings, the colored boxes represent the interquartile ranges, and the black vertical lines represent the medians. The statistically significantly differing pairs $(p<0.05)$ found when changing only one parameter (camera height $(C H)$, actor behavior $(A B)$, viewer position (VP)) are highlighted with linking lines.

The participants were $20-52$ years old $(\mathrm{M}=27.3, \mathrm{SD}=9.28)$, and $157-189 \mathrm{~cm}$ tall $(\mathrm{M}=175.8, \mathrm{SD}=8.05) .8$ of the participants were wearing eyeglasses and 2 contact lenses at the time of the experiment. 17 of them studied computer science, and 7 studied other fields. The self-reported English skills were basics for 1, good for 16, and fluent for 7 of the participants. Only 3 participants had watched $360^{\circ}$ videos several times while almost half (11) had watched them a couple of times at most. The rest had only heard/read about them (6) or had no experience at all (4). Earlier experience with virtual reality technologies in general was also rather limited: Only 1 had used such technologies a lot, 7 a couple of times at most and the rest had even less or had no experience on VR technologies, such as VR glasses. The participants did not experience fear of small or enclosed spaces to a noteworthy extent: 14 reported not to experience such fear at all, and 10 a little. When asking about experiencing nervousness or negative feelings in social situations, 9 answered not at all, 13 a little and 2 somewhat.

\section{Results}

We first present the experience results from each of the 24 conditions and analyze the effect of viewers' own height towards these experiences. Then, we present the results from the camera height preference selections. Referring to our research questions, we hypothesize that all three independent variables influence the viewing experience, and also that the viewer's height correlates with their preferred camera heights.

\subsection{Viewing Experiences}

\subsubsection{User Experience per Condition}

To find out whether our varied parameters affect the viewing experience of $360^{\circ}$ videos, we investigated if there are statistically significant differences in the user ratings between the 24 conditions. These conditions were different combinations of the following:

1) camera height $(\mathbf{C H})$ with four levels (height 1, 2, 3, 4, i.e., $105 \mathrm{~cm}, 132 \mathrm{~cm}, 159 \mathrm{~cm}$, and $185 \mathrm{~cm})$,

2) actor behavior (AB) with three levels (away, movement, near),

3) viewer position (VP) with two levels (sitting, standing)

The overview of the condition-specific user experience results regarding the naturalness and presence can be seen in Figure 5.

As our data were based on several repeated measures and of ordinal scale, and also, not normally distributed, non-parametric Friedman's test was used. Statistically significant $(p<0.05)$ differences between the conditions were found for both user experience statements. As the 24 conditions result in 276 different condition pairs, and 54 statistically significantly differing pairs for naturalness and 11 for presence, a thorough coverage of the results is out of the scope of this paper. Thus, we concentrate on reporting one-way interactions, and leave out complex two-way and three-way interactions. The reported differing pairs are also highlighted in Figure 5. 
Watching the people felt natural. Investigating the effect of camera height on the naturalness, we discovered four differing pairs. For sitting-away, watching the people felt more natural with the camera height 2 compared to height 4 . For standingmovement, camera height 3 was more natural than height 1 . In addition, for standing-near, camera height 3 was more natural than heights 1 and 2. The same effect was not observed for height 4 indicating that the highest camera position at $185 \mathrm{~cm}$ may have been too high on average even when the participants were standing.

Considering actor behavior, for sitting-height 1 the participants unsurprisingly felt less natural to watch the people when the actors were near compared to them being away. Finally, considering viewer position, for movement-height 1 , sitting was rated more natural than standing.

I felt like I was actually in the space. The presence statement experiences revealed only one statistically significantly differing pair when investigating the effect of changing one parameter at a time: Considering camera height, for sitting-away, the participants felt more as if they were actually in the space with camera height 2 than with height 4 . This same difference was found also for naturalness, as stated earlier.

Overall, the condition sitting-away-height 2 was rated the highest in terms of both naturalness and presence with medians 5 and 5.5 out of the maximum 7. Only a few participants ( 1 and 2 out of 24) rated this condition below the neutral level, i.e., neither agree nor disagree, while $75 \%$ and $79 \%$ of the participants were on the agreeing side. For naturalness of watching the people, the least agreed condition was standing-near-height 1 with a very low median of 2. For the feeling of actually being in the space, the lowest-ranked condition, in turn, was sitting-near-height 4 with a median of 3.5 .

We also investigated the relationship between the participants' background information and the experience results, and found one effect worth mentioning: prior experience with $360^{\circ}$ videos seemed to slightly disturb the illusion of actually being in the space $(5 / 24$ conditions with an $\mathrm{r}-0.41--0.523, \mathrm{p}<0.05)$.

\subsubsection{The Effect of Viewer Height}

Contrary to our expectations, participants' own height had very little effect on the user experiences between the conditions. Only two statistically significant $(p<0.05)$ correlations were found for both statements, naturalness and presence. Considering naturalness, sitting-movement-height 1 was rated slightly lower the taller the participants were themselves $(\mathrm{r}=-0.483)$. On the other hand, for standing-near-height 3 watching the people felt somewhat more natural for the taller participants $(\mathrm{r}=0.658)$. Also, for the same condition there was a weak positive correlation $(r=0.483)$ between participants' own height and how strongly they felt like being in the space. A similar weak effect $(\mathrm{r}=0.457)$ was found for sitting-near-height 2 .

\subsection{Camera Height Preferences}

We investigated the comfortable camera height range in relation to viewer position (sitting or standing) and actor behavior (away, movement, or near). For all six conditions, participants chose their most comfortable camera height, as well as the highest and lowest camera heights that they deemed still comfortable to them. The average height preference selections for different situations are represented in Figure 6. The most comfortable heights averaged $137-158 \mathrm{~cm}$ depending on the condition, whereas the highest still comfortable values were $159-180 \mathrm{~cm}$, and the lowest still comfortable values were $110-142 \mathrm{~cm}$ on average.

Surprisingly, our data revealed no statistically significant correlations between participants' self-reported height and their camera height preferences.

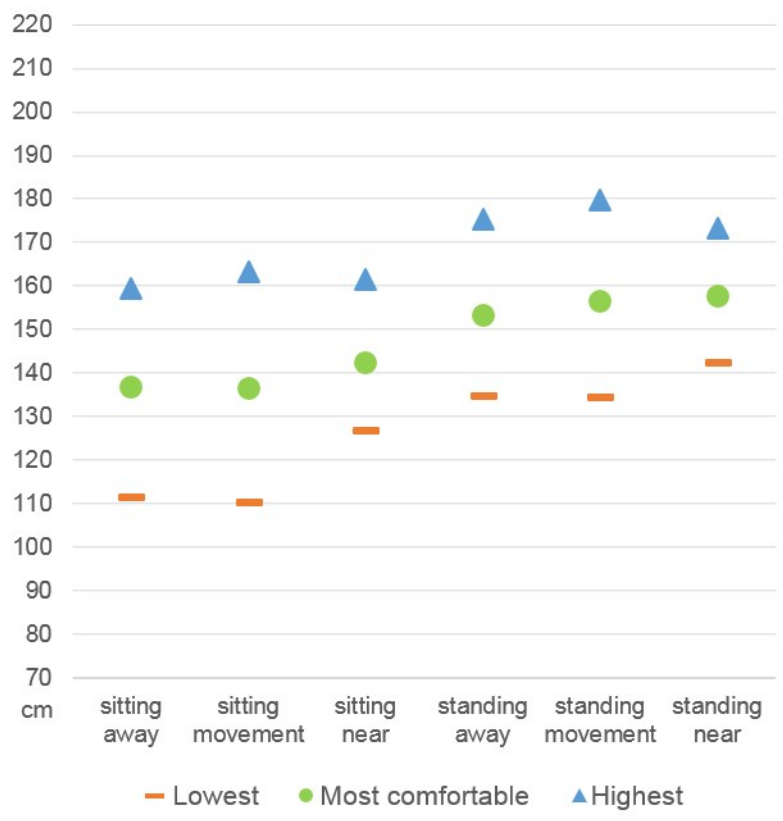

Figure 6: The average preferences for the most comfortable, and the lowest and the highest still comfortable, camera heights in relation to viewer position and actor behavior.

When looking at the most comfortable heights between the six camera height preference conditions, according to Friedman's test there are statistically significant $(\mathrm{p}<0.05)$ differences only in 4 pairs out of the 15 possible pairs. All the differing pairs have a link between sitting and standing - selected heights for sitting being lower, non-surprisingly - but only one of the pairs deals with the same actor behavior, movement.

Friedman's test further showed that there were statistically significant $(p<0.05)$ differences only for three pairs out of the 15 possible pairs when looking at the highest still comfortable heights. Again, the differing pairs relate to viewer position, that is, differences between sitting and standing.

When looking at the lowest still comfortable heights, eight pairs out of the 15 possible pairs showed statistically significant $(p<0.05)$ differences. Apart from one pair, sitting-away and sitting-near (away<near), the differing pairs relate to viewer position (sitting versus standing).

\section{Discussion and Conclusion}

Here, we discuss our findings and their implications, primarily with regards to camera height and actor behavior in $360^{\circ}$ videos. We conclude with a summary of our main findings, and present pointers for future work.

\subsection{Camera Height in $360^{\circ}$ Videos}

Out of the four tested camera heights $(105,132,159,185 \mathrm{~cm})$, the best camera height for sitting was $132 \mathrm{~cm}$, and $159 \mathrm{~cm}$ for standing, both in terms of naturalness and the sense of presence. Overall, $132 \mathrm{~cm}$ has a very slight edge in naturalness, and $159 \mathrm{~cm}$ has a very slight edge in presence. In short, the two heights (132 and $159 \mathrm{~cm})$ are practically equal. The lowest and highest viewpoints $(105$ and $185 \mathrm{~cm})$ were not rated as highly, the lowest camera height being rated the worst. We found essentially no correlation between the viewer's height and their experiences about the different camera heights, as slight correlations were found only in a few rare instances. 
In addition, the participants could choose their preferred camera height, and the comfortable camera height range, amongst 16 available options $(71.5-219 \mathrm{~cm})$. For sitting viewers, the most comfortable height averaged $\sim 140 \mathrm{~cm}$, and the comfortable range was $\sim 110-163 \mathrm{~cm}$. For standing viewers, the most preferred height was $\sim 155 \mathrm{~cm}$, and the comfortable range was $\sim 134-180$ $\mathrm{cm}$. Again, there was no correlation between the participant's own height and their preferred camera heights.

The best-performing camera heights, both in terms of viewing experience as well as preferences, were much lower than the real eye level of most viewers. This is in line with another study, where users preferred camera heights that were too low over those that were too high [19]; however, the other study did not pinpoint the actual preferred heights of the camera. In our study, the preference towards lower camera heights is particularly curious because all our tested scenarios contained people, and in some cases in very close proximity. People surely work as a great point of reference for establishing a sense of height. As such, it is reasonable to assume that the participants were aware that the viewpoints they preferred were lower than their own eye level.

Our results provide a good baseline height for many types of $360^{\circ}$ content. Considering the results above, we argue that placing the camera at around $150 \mathrm{~cm}$ will hit the comfortable height range for both sitting and standing viewers, and will also situate nicely between our two tested camera heights that provided the best viewing experience.

Our results also show that there is some freedom in camera placement for $360^{\circ}$ videos, as the comfortable heights had a range of roughly 0.5 meters for both viewing positions. This is useful because many effects can be achieved by changing the camera height (referred to as camera angle in traditional filmmaking). For example, experiments in film studies have shown that the camera angle can be used to convey feelings such as trustworthiness, attractiveness [3][15], and dominance [8]. In $360^{\circ}$ videos, camera height may affect the viewer's perception of self [16][17], and may affect how content and the actors within this content are perceived [3][8][15].

\subsection{Actor Behavior in $360^{\circ}$ Videos}

The viewing experience was generally rated lower when the actors were near the camera, compared to when they were standing further away or moving around the scene. The results therefore suggest that the distance between the camera and people in the $360^{\circ}$ video is advisable to be more than 1 meter. This is in line with Hall's [7] model of proxemics, where he reported the social distance to be between $1.2 \mathrm{~m}$ and $3.7 \mathrm{~m}$. By placing the actors outside the intimate distance $(46-100 \mathrm{~cm})$ and personal distance $(46-122 \mathrm{~cm})$, designers can provide the viewer with a "buffer zone", thus avoiding the feeling of their personal space being invaded. Saarinen et al. [20] also recommended avoiding objects close to the camera in $360^{\circ}$ videos. The link to real-world behaviors [7] may also be a testament to the immersive nature of $360^{\circ}$ videos.

We also logged the participants' gaze during the study. These data were used to provide two measures: the average number of eye blinks and the average angle between gaze and the head's orientation. However, both remained somewhat inconclusive and therefore we omitted detailed analysis of the gaze data from this paper. Regarding eye-blink frequency, there were no statistically significant effects between the conditions. Regardless, some differences were detected, as the number of blinks varied between 13.5 and 20.4 per minute depending on the condition. The potential reasons behind these differences could be an interesting future research topic. For example, the connection between eye blinking and the perceived level of stress has been reported from various sources, but the results are still inconclusive, and even contradicting [5][22]. These discrepancies are discussed in more detail in [12]. Moreover, our gaze data showed that participants moved their eyes more in relation to the head when people in the video appeared close. It is possible that the participants did this to appear less conspicuous to the people facing them, suggesting that viewers may have experienced discomfort; however, additional research must be conducted to confirm this.

\subsection{Takeaways and Conclusions}

We formulate three main takeaways from our results. First, the viewer's own height seems to have no effect on their experiences nor their preferred camera heights when viewing $360^{\circ}$ content with HMDs. This is an encouraging finding, as it suggests that designers do not need to worry about the viewers' height. Second, the best viewpoints were lower than expected, as they were well below the actual eye level of the viewers. Based on the viewing experiences and camera height preferences, a camera height of roughly $150 \mathrm{~cm}$ would hit the mark for both sitting and standing viewers. Third, we found that people standing close to the camera somewhat hindered the viewing experience, and also resulted in the viewers exploring the scene by moving their eyes more compared to people being further away, perhaps as a sign of discomfort.

We believe that our results apply to many types of $360^{\circ}$ content, although it is almost certain that they do not apply universally. It is likely that camera height plays a lesser (or different) role in some types of content, for example, those that are not grounded in reality, or where the scale of the scene is difficult to assess. That being said, our experiment focused on people, and their proximity and movement, which are core aspects in a considerable number of $360^{\circ}$ experiences.

In the future, further understanding on viewing preferences might be gained by experimenting with different camera heights in unusual $360^{\circ}$ environments, for example, those of unordinary scale, or very busy environments. Moreover, large-scale utilization of gaze data may provide further insight into $360^{\circ}$ viewing behaviors.

\section{ACKNOWLEDGMENTS}

We would like to thank Claudia Buchner for participating in the design and preparations of the user study and conducting the experiments. This work was done as a part of Immersive Media Disruption (IMD) project, financed by Tekes — the Finnish Funding Agency for Innovation (later Business Finland). We thank all the participants.

\section{REFERENCES}

[1] L. Argyriou, D. Economou, V. Bouki, and I. Doumanis. Engaging immersive video consumers: Challenges regarding 360-degree gamified video applications. In Proc. IUCC-CSS, pp. 145-152. IEEE, 2016. doi: 10.1109/IUCC-CSS.2016.028

[2] J. N. Bailenson, J. Blascovich, A. C. Beall, and J. M. Loomis. Equilibrium theory revisited: Mutual gaze and personal space in virtual environments. Presence: Teleoperators \& Virtual Environments, 10(6):583-598, 2001. doi: 10.1162/105474601753272844

[3] A. M. Baranowski and H. Hecht. Effect of camera angle on perception of trust and attractiveness. Empirical Studies of the Arts, 36(1):90-100, 2018. doi: 10.1177/0276237417710762

[4] M. Gödde, F. Gabler, D. Siegmund, and A. Braun. Cinematic Narration in VR-Rethinking Film Conventions for 360 Degrees. In Proc. VAMR, pp. 184-201. Springer, Cham, 2018. doi: 10.1007/9783-319-91584-5 15

[5] M. Haak, S. Bos, S. Panic, and L. J. M. Rothkrantz. Detecting stress using eye blinks and brain activity from EEG signals. In Proc. DCII, pp. 35-60. 2009. 
[6] J. Hakulinen, T. Keskinen, V. Mäkelä, S. Saarinen, and M. Turunen. Omnidirectional video in museums - authentic, immersive and entertaining. In Proc. ACE, pp. 567-587. Springer, 2017. doi: 10.1007/978-3-319-76270-8_39

[7] E. T. Hall. The Silent Language. Doubleday, 1959.

[8] W. Huang, J. S. Olson, and G. M. Olson. Camera angle affects dominance in video-mediated communication. In Proc. CHI EA, pp. 716-717. ACM, 2002. doi: 10.1145/506443.506562

[9] P. Kallioniemi, T. Keskinen, J. Hakulinen, M. Turunen, J. Karhu, and K. Ronkainen. Effect of gender on immersion in collaborative iODV applications. In Proc. MUM, pp. 199-207. ACM, 2017. doi: $10.1145 / 3152832.3152869$

[10] P. Kallioniemi, V. Mäkelä, S. Saarinen, M. Turunen, Y. Winter, and A. Istudor. User experience and immersion of interactive omnidirectional videos in cave systems and head-mounted displays. In Proc. Interact, pp. 299-318. Springer, 2017. doi: 10.1007/978-3319-68059-0_20

[11] R. N. Kraft. The influence of camera angle on comprehension and retention of pictorial events. Memory \& cognition, 15(4):291-307, 1987. doi: $10.3758 / \mathrm{BF} 03197032$

[12] W. Liao, W. Zhang, Z. Zhu, and Q. Ji. A real-time human stress monitoring system using dynamic Bayesian network. In Proc. CVPR. IEEE, 2005. doi: 10.1109/CVPR.2005.394

[13] J. Loomis, J. Blascovich, and A. Beall. Immersive virtual environment technology as a basic research tool in psychology. Behavior Research Methods, Instruments, \& Computers, 31(4):557564, 1999. doi: 10.3758/BF03200735

[14] A. MacQuarrie and A. Steed. Cinematic virtual reality: Evaluating the effect of display type on the viewing experience for panoramic video. In Proc. VR, pp. 45-54. IEEE, 2017. doi: 10.1109/VR.2017.7892230

[15] T. A. McCain, J. Chilberg, and J. Wakshlag. The effect of camera angle on source credibility and attraction. Journal of Broadcasting \& Electronic Media, 21(1):35-46, 1977. doi: 10.1080/08838157709363815

[16] P. J. Passmore, M. Glancy, A. Philpot, A. Roscoe, A. Wood, and B. Fields. Effects of viewing condition on user experience of panoramic video. In Proc. ICAT-EGVE, Dirk Reiners, Daisuke Iwai, and Frank Steinicke (Eds.). The Eurographics Association, 2016. doi: 10.2312/egve.20161428

[17] A. Philpot, M. Glancy, P. J. Passmore, A. Wood, and B. Fields. User Experience of Panoramic Video in CAVE-like and Head Mounted Display Viewing Conditions. In Proc. TVX, pp. 65-75. ACM, 2017. doi: $10.1145 / 3077548.3077550$

[18] S. Rothe, H. Hußmann, and M. Allary. Diegetic cues for guiding the viewer in cinematic virtual reality. In Proc. VRST, Article 54, 2 pages. ACM, 2017. doi: 10.1145/3139131.3143421

[19] S. Rothe, B. Kegeles, M. Allary, and H. Hußmann. The impact of camera height in cinematic virtual reality. In Proc. VRST, Article 124, 2 pages, Stephen N. Spencer (Ed.). ACM, 2018. doi: $10.1145 / 3281505.3283383$

[20] S. Saarinen, V. Mäkelä, P. Kallioniemi, J. Hakulinen, and M. Turunen. Guidelines for designing interactive omnidirectional video applications. In Proc. Interact, pp. 263-272. Springer, 2017. doi: 10.1007/978-3-319-68059-0_17

[21] A. Tse, C. Jennett, J. Moore, Z. Watson, J. Rigby, and A. L. Cox. Was I There?: Impact of Platform and Headphones on 360 Video Immersion. In Proc. CHI EA, pp. 2967-2974. ACM, 2017. doi: $10.1145 / 3027063.3053225$

[22] B. Zheng, X. Jiang, G. Tien, A. Meneghetti, O. N. M. Panton, and M. S. Atkins. Workload assessment of surgeons: correlation between NASA TLX and blinks. Surgical endoscopy, 26(10), 2746-2750, 2012. doi: 10.1007/s00464-012-2268-6 